Mots. Les langages du politique

\title{
Effets d'oralité dans la presse engagée : dialogisation et idéologisation du discours
}

Orality effects in the committed press: dialogisation and ideologisation of the discourse

Efectos de oralidad en la prensa comprometida: dialogisación et idéologisación

del discurso

\section{Annabelle Seoane}

\section{grevues.org}

Édition électronique

URL : http://mots.revues.org/22851

DOI : $10.4000 /$ mots.22851

ISSN : 1960-6001

\section{Éditeur}

ENS Éditions

\section{Édition imprimée}

Date de publication : 10 juillet 2017

Pagination : 167-189

ISSN : 0243-6450

Distribution électronique Cairn

\section{CAIRN INFO}

CHERCHER, REPÉRER, AVANCER.

Référence électronique

Annabelle Seoane, «Effets d'oralité dans la presse engagée : dialogisation et idéologisation du discours », Mots. Les langages du politique [En ligne], 114 | 2017, mis en ligne le 10 juillet 2019, consulté le 24 juillet 2017. URL : http://mots.revues.org/22851 ; DOI : 10.4000/mots.22851 


\section{Effets d'oralité dans la presse engagée : dialogisation et idéologisation du discours}

Dans une démarche d'analyse du discours, nous nous intéresserons à la façon dont certains titres de la presse écrite ont recours à de nombreux effets d'oralité pour se poser dans le champ médiatique comme des journaux indépendants et engagés. Nous prendrons à cet égard deux journaux qui optent pour une posture de dénonciation : Le Canard enchaîné : journal satirique paraissant le mercredi dans ses numéros de 2015, et Minute : hebdomadaire politiquement incorrect, dans ses articles en ligne depuis 2013 ${ }^{1}$. Le choix de ces deux titres se place dans une perspective contrastive, entre deux hebdomadaires qui revendiquent le rejet du politiquement correct : l'un, Le Canard enchaîné, caractérisé par Martin (2003) comme un "objet politique mal identifié »² et l'autre, Minute, un journal qualifié d' "extrême droite ", par la plupart des autres titres de presse grand public 3 - «le généraliste proche de l'extrême droite, qui se veut rassembleur de la droite souverainiste et nationaliste »4, comme par Alice Krieg-Planque (1999) ou Simone Bonnafous et Malika Temmar (2008, p. 62-63).

Ce corpus sera étudié selon différentes perspectives : linguistique, énonciative et pragmatique, sociodiscursive. Les articles cités ont été retenus en fonction de leur représentativité eu égard à la prégnance des effets d’oralité

1. Pour des raisons d'échantillonnage suffisamment large et d'accessibilité du corpus, nous avons dû élargir la période de recueil pour Minute (2013-2015 contre 2015 pour Le Canard enchaînê).

2. «Sa ligne politique a fait l'objet d'un débat depuis sa fondation en 1915, plus vif toutefois parmi les historiens et politologues qu'au sein de la rédaction. Si l'image d'un journal anarchisant ressort de la majorité des analyses, d'autres mettent l'accent sur le républicanisme du Canard ou bien sur ses liens avec le communisme» (Martin, 2003, p.73), même si «aujourd'hui, si cette sensibilité n'a pas disparu, elle s'est notablement estompée. Depuis les années soixante, la logique militante a eu tendance à céder le pas à la logique de l'information ; la montée en puissance, dans les colonnes du Canard, de ce qu'il est convenu d'appeler le “journalisme d'investigation" a entraîné dans son sillage un désengagement (plutôt qu'une dépolitisation) de l'hebdomadaire satirique» (Martin, 2003, p. 90-91).

3. L'Express, 12 novembre 2013; Le Figaro, 14 novembre 2013; La Voix du Nord, 12 novembre 2013; www. francetvinfo.fr et Agence France-Presse, 7 janvier 2013; Europe 1, 20 novembre 2014, entre autres.

4. C. De Malet, «Minute en liquidation judiciaire », Le Figaro, 13 novembre 2013 〈http://www.lefigaro.fr/politique/2013/11/13/01002-20131113ARTFIG00627-minute-un-journal-qui-vit-de-sesprovocations.php> (consulté le 11 octobre 2016).

Université de Lorraine, Centre de recherches sur les médiations : communication, langue, arts, culture (EA 3476)

annabelle.seoane@univ-lorraine.fr

Mots. Les langages du politique $\mathrm{n}^{\circ} 114$ juillet $2017 \bullet 167$ 
que nous souhaitions analyser. Nous traiterons ici non pas des traits d'oralité (comme l'insertion de discours cités) mais bien d'effets d'oralité dans certains discours de presse. Nous entendons par là des procédés habituellement confinés à des pratiques de communication orale, indices d'une représentation sélective de l'oralité dans l'écriture (Gadet, 1991), "selon l'angle de la restitution-figuration de l'oral dans l'écrit où toute monstration de l'oral est nécessairement du pseudo-oral» (Rosier, 2000, p. 26).

D’un point de vue méthodologique, nous aborderons en premier lieu les procédés qui relèvent de ces effets d'oralité au niveau local, microtextuel, puis nous en analyserons les incidences énonciatives et pragmatiques au niveau global, macrotextuel, axées sur la mise en scène d'une conflictualité latente ou explicite. Après avoir passé en revue les principaux procédés d'oralité mis en œuvre, lexicaux, syntaxiques et discursifs, il s'agira de montrer que ces locuteurs journalistiques déploient par là-même une stratégie pragmatique double, que l'on pourrait résumer par la volonté de sortir du rang d'une part et d'autre part, celle de former communauté5. Cette stratégie apparaît en lien avec la notion de connivence. Ces discours optent en effet pour une posture critique qui vise à accomplir un acte de langage de dénonciation et la construction d'un ethos de journalisme libre de toute forme d'assujettissement politicomédiatique.

Nous partons donc de l'hypothèse que les journaux engagés n'ont pas recours à des effets d'oralité comme de simples variations stylistiques (Chaput, 2009), mais comme des indicateurs d'une dynamique pragmatique et d'idéologisation du discours, ces procédés reposant sur une stratégie de fonctionnement en décalage et sur un rapport à l'autre qui table sur la capacité (ré) interprétative du lecteur. Cette stratégie discursive dynamique est soutenue par une scénographie qui construit un ethos de journaliste impertinent et indépendant par le biais d'un univers de discours aux singularités langagières prégnantes et tout particulièrement à travers des effets d'oralité conçus comme des micro-transgressions des codes routiniers de la presse écrite. Le discours répond ainsi aux contraintes de son genre, mais construit également de nouveaux codes identificateurs et par là-même une communauté discursive.

S’il n'existe pas de structures linguistiques spécifiques à l'oral, la récurrence de certains traits peut témoigner d'une orientation plus «orale» ou «écrite » du discours, comme le fait remarquer Françoise Gadet (1991, p. 110111) : «il existe un sous-ensemble de phénomènes pour lesquels intervient non une différence de fonctionnement, mais la fréquence d'une forme ou d'une autre selon le mode de transmission. » Claire Blanche-Benveniste (1996, p. 117) observe également dans ses travaux cette intrication : «Faire une nette séparation entre grammaire de l'écrit et grammaire de l'oral ne paraît pas s'imposer

5. Voir Bayle, Bombart, Garnier et Boisséras, 2016. 
[...] II me paraît important de voir comment, dans tous ces problèmes d'ordre des mots, la syntaxe, la sémantique et l'intonation sont liées. " Sans être trop dichotomique, la distinction oral/écrit se joue alors sur la fréquence de telle ou telle forme et découle généralement des conditions d'utilisation car l'oral repose sur un principe d'immédiateté et d'ancrage dans la situation d'énonciation et de réception, entraînant toute une série de procédés linguistiques et discursifs en lien avec l'échange communicatif en lui-même (économie des moyens linguistiques, interaction possible de l'allocutaire...). L'insertion de ces effets d'oralité dans le quotidien produit un effet d'écrit (presque) spontané selon l'axe de variation du français parlé (Blanche-Benveniste, 1997).

L'oscillation oral/écrit pose ainsi à certains égards la question des rapports entre formel et non formel et interroge les routines du champ de l'écrit journalistique, le rapport à la norme et à l'institution. Cette réflexion sur l'investissement locutoire ou illocutoire du locuteur-journaliste ne suggère pas des rapprochements tant hâtifs que simplistes entre l'écrit qui serait par nature neutre et conventionnel et l'oral, familier et révélateur, mais tâche plutôt de démontrer la récurrence de ces effets d' «hétérogénéité énonciative» qui peuvent fonctionner comme des outils de dialogisation, des indices de parler vrai dans un corpus donné.

\section{Procédés d'oralité mis en œuvre}

Ces dispositifs qui imitent des traits d'oralité en reproduisant une illusion de verbalisation entrent donc dans cette stratégie du dire. Ils se manifestent dans certaines tournures lexicales, syntaxiques, morphosyntaxiques ou rythmiques. Le discours tangue entre standards d'une écriture de presse écrite et effets d'oralité.

\section{Une énonciation particulière}

Relevons pour commencer certaines constructions syntaxiques plus courantes à l'oral : l'élision du «ne» dans la négation, des procédés de mise en relief comme certaines tournures complétives focalisantes propres à l'oral, les détachements, les dislocations et antépositions de certains compléments à des fins de topicalisation (Blanche-Benveniste, 1996, p.117), la prévalence de la tournure présentative en «c'est» (dispositifs de clivage en «c'est... que...»), car, comme le souligne F. Gadet (1991, p.112) :

De l'oral, on ne retient que l'usage populaire [...], c'est le jaillissement spontané [...], tendanciellement dépourvu de règles, l'oral est un lieu où tout est permis [...] Les détachements sont donc tendanciellement considérés comme oraux, familiers, populaires, spontanés, échappant aux règles et expressifs. 
Deux ans, c'est un bel âge pour voter radical. (C. E., 18 juin 2014)

Un peloton d'exécution, c'est trop demander? (M., 7 mai 2013)

Pour une fois qu'il ne s'est pas pris des trombes d'eau sur la tronche, il faut s'estimer heureux. On commençait à être habitué à le voir tout dégoulinant, notre président. (M., 2 juin 2015)

Par son lien avec l'oralité, le détachement droit suppose que le référent a déjà été identifié comme tel par les partenaires de l'interlocution et fait entendre une autre voix, fait écho ironiquement (Sperber et Wilson, 1978) à une autre parole, et peut être considéré comme un «fonctionnement d'écho ironique dialogique » (Nowakowska, 2009, p. 84).

On note également de nombreuses répétitions car «l’oral est généralement interprété comme le domaine des réitérations de formes ou de schémas syntaxiques identiques. En dehors des phénomènes de bribes liés au mode de production, il est vrai que les locuteurs produisent souvent des répétitions de mêmes éléments dans des positions syntaxiques identiques, mais il n'est pas toujours aisé de [leur] donner un statut; ce peut être un procédé d'insistance [ou pour produire] entre autres, un effet de contraste » (Blanche-Benveniste et Bilger, 2009) :

Non, non, l'UMP n'est pas morte... (C. E., 3 juin 2015)

Fait maison mais oui mais non. (C. E., 13 mai 2015)

Parce que la France, c'est beau, c'est grand, c'est généreux, et que ça se refuse à séparer un Ahmed de sa maman et de ses frères et sœurs. (M., 30 avril 2015)

Mais que diable la France lui a-t-elle donc fait? En fait, rien. Rien, si ce n'est du bien. (M., 30 avril 2015)

Ces procédés relèvent également de la prosodie. Le groupe rythmique, unité fondamentale de l'oral, est retranscrit à l'écrit par des jeux sur le séquençage phrastique ou syntagmatique pour reproduire le rythme et la prosodie qu'aurait l'oral, avec des incidences syntaxiques et lexicales. Ces regroupements ne sont pas toujours signalés par des morphèmes ou par des tournures syntaxiques, entraînant un rythme paratactique, des phrases en suspens, averbales ou inachevées, avec un emploi massif de la coordination « et » en tête de phrase :

Moralité : pour que les sociétés d'autoroute arrêtent de se gaver sur son dos, l'État commencera par gaver un cabinet d'avocats conseil. (C. E., 11 février 2015)

En 2020, peut-être? (C. E., 24 juin 2015).

Et cet énergique magistrat a poussé le talent encore plus loin. (C. E., 10 juin 2015)

Encore une promesse qui a fait pschitt!6 (M., 19 mars 2013)

Et cette fois, le retrait en rase campagne a été prestement mené. (M, 15 mars 2016)

6. Allusion probable à la déclaration de Jacques Chirac, alors Président de la République, le 14 juillet 2001, visant à minimiser l'affaire du financement occulte de ses voyages. 


\section{Une énonciation hybride}

Ces procédés se trouvent régulièrement combinés à des procédés lexicaux : - des mots issus d'un registre familier ou argotique, utilisé habituellement plus volontiers à l'oral :

Surtout quand l'ex ne cesse de donner des leçons de bonne gestion aux socialos... (C. E., 3 juin 2015)

Le Refuge, une association pour homos très chouchoutée [...] qui est cul et chemise avec Taubira. (M., 24 février 2015)

- avec des réduplications possibles:

Dans le cas de fifille, il ne s'agit pas de gros millions et de lingots planqués en Suisse. (C. E., 27 janvier 2016)

- des mots d'enfant:

La légende veut que Carla Bruni-Sarkozy ait manqué de défaillir, à l'été 2008, en voyant du caca suinter d'un rocher. (C. E., 10 juin 2015)

Des familles entières, avec papa-maman, papy-mamie. (C. E., 18 juin 2015)

Il a dix ans quand il arrive en France avec sa maman pour y retrouver son papa. (M., 30 avril 2015)

- des dislocations du type : «Tout le monde il est content (M., 2 février 2015)7

- l'utilisation de formes empruntées à des domaines sociodiscursifs spécifiques:

José Bové, sors de ce pape! (C. E., 24 juin 2015)

Bravo, maître Sarko! (C. E., 10 juin 2015)

Esprit, es-tu toujours là? (M., 30 avril 2015)

Qu'on les fusille! (M., 7 mai 2013)

Ces procédés fréquents sont rentables du point de vue pragmatique : ils jouent de la représentativité latente d'un «signum social» en fonctionnant comme des signaux de transgression d'un cadre normatif journalistique, ouvrant sur un espace énonciatif autre, que le lecteur est amené à devoir

7. «La tournure de dislocation, que les grammaires appellent parfois "redondance syntaxique" (mon père, il arrive) a été attribuée tour à tour au manque de maturité enfantine, aux influences étrangères, aux régions, ou à une évolution récente de la langue [...] Tout le monde il a fait ça se signale au contraire comme particularité qui déclasse (“signum social”, disent certains)» (Blanche-Benveniste, 2010, p. 37). 
identifier. L'effet de décalage induit une tonalité sarcastique qui produit une mécanique interlocutive de connivence (Seoane, 2015, p.102).

\section{Une énonciation polarisée}

D’autres procédés relèvent d'aspects morphosyntaxiques et syntaxiques, avec, notamment, la présence d'exclamatives propres à l'oral et d'interjections :

«Mon œil! (C. E., 3 juin 2015)

Quelle pitié ! [...] Futé! (C. E., 11 février 2015)

Vive la bouffe aseptisée! (C. E., 17 juin 2015)

Hélas, des rabat-joie sont venus gâcher l'ambiance. (C. E., 17 juin 2015)

Eh ben, c'est pour éviter les recours. (C. E., 27 janvier 2016)

Ça alors! (M., 30 avril 2015)

Car ce vieux pays en a connu d'autres, bon Dieu! (M., 14 octobre 2015)

Je vous demande un peu! (M., 14 octobre 2015)

Hélas, deux ans plus tard, il est contraint d'écourter son séjour faute de papiers en règle. ( $M ., 30$ avril 2015)

Ouf, on a eu peur! (M., 7 mai 2013)

Allez les djeunes, bougez-vous, la gérontocratie a besoin de vous! ( $M ., 22$ décembre 2015)

Ces exclamatives prototypiques ressortent d'une prise en charge axiologisée explicite (de contentement, de soulagement, de dépit...), presque théâtralisée, faisant écho plus ou moins ironiquement à des positionnements extérieurs mis à distance.

L'exclamation peut être accompagnée par l'ellipse du «il » impersonnel ou par l'emploi du pronom démonstratif «ça» - plus fréquents dans la pratique orale, comme le mentionne Peeters (2007):

Ça y est! [...] On se fait un restau maison ou on reste à la maison? (C. E., 13 mai 2015)

Tiens! y'a plus d'agressions contre les homos? (M., 7 mai 2013)

D'autres exclamatives marquent l'attitude de l'énonciateur par rapport à son dire, comme dans les bribes autocorrectives introduites par « euh... » :

Le ministère de l'Économie n'a trouvé personne dans ses maigres effectifs - 145000 fonctionnaires! - pour remplir cette mission hautement, euh... économique. (C. E., 11 février 2015)

Et toc! Mais cette race, euh... je veux dire ces gens-là, ont toutes les audaces. (M., 30 avril 2015) 
Ce micro-dispositif est double puisque l'onomatopée d'hésitation «euh » est appuyée par les points suspensifs reproduisant à l'écrit la pause qui serait faite à oral. L'énoncé donne l'image d'un énonciateur cherchant l'euphémisme, le plus approprié ou corrigeant son propos pour se conformer au politiquement correct : l'euphémisme se donne alors pour tel et dénonce le politiquement correct, dont le journaliste se distancie au nom du parler vrai. L'exclamation contribue à marquer cette mise à distance et à lui donner une valeur de suspicion : le positionnement du journal ne se situe pas simplement dans la distanciation objectivante, il est nettement expressif, le but n'est pas seulement d'informer mais de dénoncer. L'effet d'oralité vient ici renforcer le dédoublement pragmatico-énonciatif en esquissant une tension entre dit et non-dit et une dualité énonciative, dialogisée et polarisée.

\section{Mises en œuvre en discours de ces effets d'oralité}

Ces procédés constituent ainsi des lieux propices pour travailler le rapport entre texte et contexte par la mise en œuvre de ces effets d'oralité indexés sur un fonctionnement en discours.

\section{Discontinuité discursive d'une énonciation dialogisée}

Au creux même de la texture énonciative de ces textes s'élaborent certains rôles énonciatifs et certaines représentations, qui affleurent dans des fonctionnements dialogiques dont les effets d'oralité peuvent constituer de bons indices. Ils offrent au locuteur-scripteur journaliste le moyen de signaler du déjà-dit ou du dit par un autre et donc de mettre en scène le discours d'un autre que lui : dire d'enfant, dire d'un «personnage » (Perrin, 2009), dire doxique (dialogisme interdiscursif), discours censé être tenu par le lecteur (dialogisme interlocutif) ou représentation d'une oralité connotée comme familière ou populaire ${ }^{8}$. Cette hétérogénéité énonciative émaille le discours et fait émerger une tension entre registres de l'écrit ou de l'oral. En posant l'intégration de discours autres, décalés, les effets d'oralité contribuent alors à installer une conflictualité latente qui apparaît dans la matérialité textuelle et qui peut induire l'idée d'une conflictualité extradiscursive dans la sphère politico-médiatique.

Nous nous efforcerons de montrer que ce dialogue interne entre énoncés est mû par le travail d'une posture métadiscursive de la part du «locuteurénonciateur» (Voir Rabatel, 2006 : le journaliste est à la fois locuteur, producteur de l'énoncé et énonciateur à la source d'un «point de vue » et d'une prise en charge énonciative et modale) et que, par conséquent, l'énonciation dialogisée

8. Comme le souligne un relecteur, cette oralité suggère-t-elle des commentaires politiques tenus dans le cercle privé, tels que le lecteur pourrait les faire pour son entourage? 
par ces effets d'oralité établit une « configuration de médiation » par ces marques de «dialogisme montré» (Authier-Revuz, 1995, p. 222) et construit un ethos particulier.

De fait, dans notre corpus, les discours transverses qui se glissent dans la trame discursive fonctionnent comme autant d'arguments et donc convergent vers la mise en scène d'un éveil d'une conscience civique voire sociale. À cet égard, les effets d'oralité font ici partie de l'outillage de négociation du journaliste face à la divulgation d'une information. Ils sont les pierres angulaires d'une scénographie dans laquelle le journaliste s'arroge un rôle mobilisateur et subversif de franc-tireur, qui s'est donné une mission de dévoilement.

\section{Mise en discours d'un dialogue fictif entre le locuteur et un personnage médiatique}

\section{Étudions cet article de Minute du 30 avril 2015 (nous soulignons les passages sur lesquels porte l'analyse) :}

[1] Esprit, es-tu toujours là? [2] A défaut de faire tourner les tables, [3] Manuel Valls fait tourner les sangs. [4] Et dans Marianne, celui de Jacques Julliard9 n'a justement fait qu'un tour : "La République marche sur la tête et le gouvernement aussi », écrit l'éditorialiste. "C'est se moquer de “l'esprit du 11 janvier" que de réduire, au nom de l'antiracisme, la liberté de la presse. C'est se moquer de Charlie Hebdo que de préparer une loi qui enverra plus souvent que naguère Charlie Hebdo devant les tribunaux. C'est un peu fort de café : je n'ai pas défilé le 11 janvier dernier pour remettre en cause la grande loi républicaine de 1881 sur la presse et la liberté d'expression! Cela s'appelle l'ordre moral. Et c'est la gauche qui prétend nous ramener à l'ordre moral!». [5] Ça alors! Qui l'eût cru ? [6] Serge Lama chantait naguère "Je suis cocu, mais content!» Jacques Julliard donne plutôt dans le cocu mécontent, personnage éternel de la comédie, du boulevard et de la politique.

Vieux routier du journalisme, Julliard a pourtant gardé une naïveté touchante. [7] La gauche, liberticide? II en tombe des nues [...] Le drame de la gauche, qui la pousse sans cesse à s'ériger en gardienne du nouvel ordre moral, [8] c'est son irrésistible propension à s'autoproclamer le camp du bien. «Imaginez un instant les mêmes mesures annoncées par Sarkozy : tout ce que la France compte d'esprits libres serait aujourd'hui vent debout!», s'indigne Julliard. [9] Erreur, mon cher confrère : le manque de réaction que vous-même constatez aujourd'hui montre simplement qu'un esprit de gauche ne fait pas un esprit libre. [10] Permettez-moi d'ajouter que s'il existait encore des esprits libres dans la presse française, ils se seraient manifestés lors du procès que Mama Doc intenta, non pas à Charlie Hebdo, titre phare de la bien-pensance [11], mais à l'éternel vilain petit canard que demeure Minute, en prenant prétexte d'un jeu de mot en couverture pour fulminer une accusation de racisme que la lecture du numéro démentait. [12] Et

9. Journaliste éditorialiste au Nouvel Observateur puis à Marianne, ancien responsable syndical de la CFDT. 
si vous-même, Jacques Julliard, aviez alors protesté dans Marianne contre cette condamnation politique, sans doute entendrais-je encore mieux votre appel «républicain » - par ailleurs légitime et bienvenu - à combattre une loi liberticide au nom de la liberté d'expression.

Le discours se pose d'emblée dans le cadre d'une mise en scène de l'oralité puisqu'il débute par une interrogative incantatoire [1], propre à un champ sociodiscursif spécifique, celui du spiritisme, ici détourné à des fins politiques. Si les deux énoncés suivants viennent justifier cette entrée en matière incongrue par un jeu de ricochets lexicaux, pourrait-on dire, entre l'univers du spiritisme [1], «faire tourner les tables » [2] et « un tour de sang » [3], suscitant de la part du lecteur des sauts interprétatifs successifs (Kerbrat-Orecchioni, 1986, p. 80), c'est la conjonction « et » en prime position en [4] qui semble porter l'effet d'oralité puisqu'elle compense la rupture rythmique et thématique marquée par le point en signalant un ajout inopiné. Cette coordination interphrastique par un coordonnant habituellement intraphrastique reproduit ainsi le processus cognitif que l'on retrouve dans l'interaction orale, celui d'un ajout après coup, dans le cadre d'un échange spontané. Ces énoncés introduits par «Et» en tête de phrase sont extrêmement fréquents dans notre corpus, particulièrement dans Minute. Pour reprendre les analyses d'Anne Abeillé (2005), les ajouts en extraposition d'un syntagme donnent au syntagme-énoncé conjoint une valeur sémantique emphatique, avec une prosodie incidente non intégrée à la prosodie du reste de la phrase.

S’ensuivent plusieurs énoncés rapportés au discours direct qui reposent sur une répétition de tournures en "c'est» : ils se placent sur une dynamique argumentative mais cet élan est cassé net par deux énoncés très brefs ("Ça alors! Qui l'eût cru? » [5]). La rupture rythmico-syntaxique signale ici un changement d'énonciateur. Ensuite, un jeu de mots en [6] entre les paroles de la chanson «Je suis cocu, mais content!» et le groupe nominal «le cocu mécontent» souligne le passage à un dispositif où les effets d'oralité marquent une axiologisation du discours. Cette axiologisation devient idéologisation avec l'interrogative averbale «La gauche, liberticide? " [7] et son métacommentaire intégrant une nouvelle structure de clivage en «c'est» [8]. A partir de là, le discours déploie en [9], [10], [12] une fiction de joute verbale entre les deux énonciateurs mis en place et opposés idéologiquement. Les effets d'oralité fonctionnent comme des modulations argumentatives et structurent le discours non seulement pragmatiquement mais aussi argumentativement, pour poser le journal comme un «éternel vilain petit canard» [11].

De nombreux articles de Minute déploient ainsi une scénographie de dialogue fictif avec un personnage médiatique et politique, parfois dès le titre : «Marine ${ }^{10}$, réveille-toi, il est devenu fou!» (23 janvier 2015), «Les Noirs ne

10. Marine Le Pen, présidente du Front national. 
sont pas tout blancs. Pardon Christiane ${ }^{11}$ !» (12 novembre 2014), parfois dans le corps du texte :

L'opération s'est pourtant révélée être un retentissant «fail» (un échec) comme on dit chez les jeunes férus d'internet, car Najat ${ }^{12}$ s'est vraiment plantée. En réponse, des centaines de tweet ont fait remarquer au ministre (oui Najat, je fais exprès de ne pas écrire «à la ministre ») qu'il avait beau jeu de venir jouer la culpabilisation des patriotes dix-neuf ans après ce drame quand, dans le même temps, elle reste silencieuse sur les actes anti-Blancs ou anti-Français. (6 mai 2014)

D’autres articles s’appuient sur des énoncés au discours indirect libre faisant intervenir le discours censé, sans doute, avoir été celui de l'intéressé pour mettre en scène le dialogue fictif. Ainsi toujours dans Minute (14 avril 2015, «Crise au Front National ») :

Dans la crise qui secoue actuellement le Front national, Florian Philippot a mis de l'huile sur le feu. Et même de l'huile sur l'huile déjà bouillante. L'occasion est trop belle de se débarrasser de Jean-Marie Le Pen. Il s'est juste montré un peu trop insistant. Et ça s'est vu. Le talon d'Achille de Florian Philippot, c'est qu'il est pressé. Très pressé. Et très sûr de lui. Sûr de ses analyses et de sa stratégie. Sûr aussi de l'ascendance qu'il exerce sur Marine Le Pen. [1] Avant lui, c'est à peine si le Front national existait. Depuis lui, le parti se dirige vers le pouvoir, Marine Le Pen vers l'Élysée et lui vers Matignon. La Constitution est mal faite qui ne prévoit pas qu'il puisse y avoir deux présidents de la République en même temps...

Ici, tout un pan de discours relève du discours indirect libre (souligné en pointillés), attribuable à Florian Philippot, vice-président du FN et conseiller de Marine Le Pen. Le locuteur-énonciateur du journal produit un commentaire sur ce discours indirect libre et simule l'adhésion à ce discours virtuel mais certains indices laissent entrevoir un décalage : en amont, certains procédés évoqués induisent des effets d'oralité par des tournures d'emphatisation, un rythme syntaxique paratactique («c’est qu’il est pressé. Très pressé. Et très sûr de lui ») ou la prime position de « et »; en aval, les points de suspension, marqueurs qui assoient la «dynamique interlocutive, en impliquant un dire in absentia qu'il appartient au lecteur de compléter» (Dahlet, 2003, p.94). Cet effet de décalage induit une tonalité sarcastique qui table sur une mécanique interlocutive de connivence. Le dernier énoncé (soulignement double) est hybride puisqu'il peut s'interpréter comme du discours indirect libre (F. Philippot qui se vanterait) ou comme un métacommentaire du locuteur-journaliste. Le lecteur bascule ainsi sans arrêt d'un discours donné comme officiel vers un discours officieux, supposé dévoiler la vérité derrière le masque du politiquement correct des personnages politico-médiatiques incriminés.

11. Christiane Taubira, Garde des Sceaux, ministre de la Justice de mai 2012 à janvier 2016.

12. Najat Vallaud-Belkacem, ministre de l'Éducation nationale, de l'Enseignement supérieur et de la Recherche depuis mai 2014. 
Tout comme A. Krieg-Planque l'a montré pour l'utilisation de «sic» dans la presse d'extrême droite, les effets d'oralité permettent alors, en discours, de «dénoncer des contre-vérités» (Krieg-Planque, 1999, p. 23-24):

L'extrême droite tourne en ridicule ce discours qui lui est extérieur (ridicule parce qu'extravagant, invraisemblable, délirant peut-être), mais aussi elle représente ce discours comme caractérisé par le mensonge et la tromperie. Et, par différence, elle se construit comme seule parole vraie. On assiste ici à une auto-représentation du discours comme seul « discours vrai », adéquat à la « réalité», auto-représentation que l'on retrouve sur des modes divers dans le discours de l'extrême droite en son ensemble. On observe aussi que le mensonge des autres est tendanciellement dirigé directement contre elle. C'est un mensonge pernicieux, énoncé avec l'intention de nuire [...] À leur modeste niveau, certains des «sic» mis en place dans les colonnes de la presse d'extrême droite participent directement à cette dénonciation du complot orchestré contre elle.

Il nous semble à cet égard que certains de ces effets d'oralité relèvent ici de la même dynamique d' «auto-représentation », notamment lorsque l'énonciation est explicitement portée par un «je » : «Permettez-moi d'ajouter» dans Minute du 30 avril 2015 évoquant Jacques Julliard; un « confrère » journaliste, «Je vous demande un peu!» (14 octobre 2015); «En réponse, des centaines de tweet ont fait remarquer au ministre (oui Najat, je fais exprès de ne pas écrire “à la ministre)" » (6 mai 2014). Cette auto-représentation se réalise aussi implicitement puisque certains procédés d'oralité ont trait à des personnages médiatiques ou des thèmes d'actualité avec lesquels préexiste une polémique avec le journal. C'est le cas notamment avec le Front National ${ }^{13}$ : « Marine, réveille-toi, il est devenu fou! », (Minute, 23 janvier 2015); ou avec Christiane Taubira ${ }^{14}$ : «Les Noirs ne sont pas tout blancs. Pardon Christiane!» (Minute, 12 novembre 2014).

13. "Mariage des homos: le torchon brûle entre la direction du FN et le journal Minute», www. francetvinfo.fr, 8 janvier 2013 〈http://www.francetvinfo.fr/societe/mariage/ mariage-et-homoparentalite/mariage-des-homos-le-torchon-brule-entre-la-directiondu-fn-et-le-journal-minute_199367.html»; «Au FN, des cadres et des élus ne veulent plus recevoir Minute », www.lepoint.fr, 27 janvier 2016 <http://www.lepoint.fr/politique/des-eluset-des-cadres-du-front-national-ne-veulent-plus-recevoir-l-hebdomadaire-d-extreme-droiteminute-27-01-2016-2013352_20.php»; «Minute - FN : un divorce étalé au grand jour qui ne date pas d'hier», www.huffingtonpost.fr, 13 novembre 2013 <http://www.huffingtonpost. $\mathrm{fr} / 2013 / 11 / 13 /$ minute-fn-divorce-etale-grand-jour-date-pas-hier_n_4265200.html> (liens consultés le 18 octobre 2016).

14. «Minute condamné en appel pour avoir comparé Christiane Taubira à un singe », www.lemonde. fr, 30 octobre 2014 〈http://www.lemonde.fr/societe/article/2014/10/30/10-0oo-euros-damende-pour-le-directeur-de-minute-ayant-compare-taubira-a-un-singe_4515241_3224. html?xtmc=taubira_minute\&xtcr=1〉; " "Taubira retrouve la banane" : le directeur de Minute condamné pour sa Une», www.lexpress.fr avec AFP, publié le 30 octobre 2014, 〈http:// www.lexpress.fr/actualite/politique/taubira-retrouve-la-banane-le-directeur-de-minutecondamne_1617093.html»; «Taubira comparée à un singe : amende de 10000 euros confirmée en appel», www.lefigaro.fr, avec AFP 17 septembre 2015 〈http://www.lefigaro.fr/ flash-actu/2015/09/17/97001-20150917FILWWWo0178-taubira-comparee-a-un-singe-a-la-unede-minute-amende-de-1000o-euros-confirmee-en-appel.php> (liens consultés le 18 octobre 2016). 
Abordons à présent le recours à ces procédés dans le Canard enchaîné, qui, moins qu'une mise en discours d'un dialogue fictif entre le locuteur et un personnage médiatique, va plutôt produire une mise en discours d'un dialogue fictif entre le locuteur et le lecteur.

\section{Mise en discours d'un dialogue fictif entre le locuteur et le lecteur}

\section{Le Canard enchaîné joue également d'une énonciation fortement dialogisée à} des fins ironiques, comme dans cet article du 18 mars 2015 intitulé «Salauds de pauvres! ». Le titre même ne peut être attribuable qu'à un personnage tiers, et non au locuteur du journal en raison essentiellement de son ethos prédiscursif (Maingueneau, 2002) :

[1] Installer 20 femmes pauvres et isolées, avec leurs enfants, au cœur d'un quartier résidentiel de sa fière bourgade? Pas question! a répondu fermement Mme la Maire [Françoise LECOUFLE, maire de Limeil-Brévannes] [...] [2] Comment? Très simple : elle a tout fait pour que les travaux ne puissent pas commencer [...] Lorsque le constructeur lui demande l'autorisation de faire passer une foreuse dans ses rues, elle décline. [3] «Pour des raisons de sécurité», argue-t-elle. [4] Pas de foreuse, pas de fondations. Et refus d'édicter le moindre arrêté municipal concernant le stationnement et le passage des camions. De manœuvre dilatoire en fin de recevoir, le chantier est arrêté. Ce qui permet à la maire de piétiner le permis de construire, avec ce motif imparable : «non-commencement des travaux». [5] Un cynisme qui confine à l'œuvre d'art. Et que le tribunal administratif devra apprécier à sa juste valeur : une action en référé a été engagée par le maître d’ouvrage, Habitats solidaires.

Le dispositif de question-réponse en début de texte met en scène un dialogue fictif entre le locuteur et le personnage médiatique (dialogisme interdiscursif : [1]), puis entre le locuteur et son lecteur (dialogisme interlocutif : [2]). Les motifs de la décision administrative sont énoncés d'abord au discours direct introduit par des guillemets [3] puis sous la forme d'une «reconstruction empathique » 15 en [4]. Cette « reconstruction empathique», par le locuteurjournaliste, des calculs censés avoir été faits par la maire pour justifier la remise en cause du permis de construire (discours intérieur reconstitué ou simulé comme tel) est repérable grâce à la syntaxe discontinue qui rappelle celle qui reproduit le rythme et la prosodie qu'aurait ici un oral saccadé, ce qui, d'un

15. "C'est ici que se greffent les processus empathiques par lesquels L1/E1 raconte, explique, argumente en envisageant les choses du point de vue d'un énonciateur second sans nécessairement lui donner la parole» (Rabatel, 2011, p. 101) ; "La notion d'émotion comme une catégorie englobant des notions connexes, humeurs, affects, sentiments, valeurs, etc. [...] Le paradoxe émotif en mode empathique revient à dire que le locuteur qui parle n'est pas celui qui prend en charge les émotions [...] L'empathie linguistique, sous son versant énonciatif, renvoie au fait qu'un locuteur prête sa voix à un autre (interlocuteur ou tiers) pour envisager un événement, une situation, à sa place.» (Rabatel, 2013, p.65-66) 
point de vue pragmatique, renforce le caractère de l'argument dénoté comme arbitraire. Le métacommentaire du locuteur-énonciateur transparaît lui aussi par la syntaxe et précisément par la position en tête de phase de la conjonction « et» en [5] : le premier syntagme se place à un niveau d'évaluation axiologique du discours par l'anaphore exophorique "cynisme » tandis que le deuxième introduit un autre niveau d'évaluation, par la justice civile. Les effets d'oralité mettent donc à jour une énonciation fortement dialogisée où se jouent des postures proprement argumentatives et dans laquelle le lecteur est partie prenante, par sa lecture oblique aussi bien que par une interlocution plus directe (tours interrogatifs).

Par le biais d'effets d'oralité omniprésents, renforcés par des marqueurs paratextuels, s'opère ici la construction d'une posture de dénonciation d'autant plus efficace qu'elle s'appuie sur une interaction dialogique qui rend opératoire la discordance entre texte et co(n)texte. Cette interlocution constitue le soubassement d'une "auto-représentation» (Krieg-Planque, 1999) qui dénonce par l'humour «tissant une connivence, fondée sur le partage de valeurs communes et une même jauge de l'acceptabilité sociale» (Seoane, 2015, p. 100).

\section{Dire par le sarcasme ou l’ironie}

Le recours à des micro-dispositifs dialogiques sert d'indice de la tension entre ce qui se dit et ce qui s'écrit et entre ce qui se dit et ne se dit pas. Une opposition surgit entre les discours de surface, de la première strate de lecture, et les discours de la deuxième strate, qui, eux, requièrent la réinterprétation du lecteur. Ces formes associables à de l'oralité permettent alors de moduler le dire «vers le haut (sarcasme) ou le bas (ironie)» (Chabrol, 2006, p. 8) en le faisant entrer dans un processus de non congruence. Le lecteur doit chercher du contenu au-delà du texte lui-même, et en cela ces indices sont des ouvertures vers un autre espace, celui de l'interdiscours ou celui l'interlocution, où la connivence contribue à construire l'ethos de dénonciateur libre du journal. Comme l'a analysé Charaudeau (2011b, p. 32) :

Sarcasme (ou raillerie) et ironie participent du même processus énonciatif dans lequel apparaît une dissociation entre le Je-locuteur porteur d'un pensé et le Jeénonciateur exprimant un dit [...] Lorsque la cible n'est pas l'interlocuteur, on retrouve l'effet de connivence, l'appel à ce que le destinataire-témoin soit complice du dénigrement, un peu comme dans la figure du commérage.

L'article du Canard enchaîné du 10 juin 2015 intitulé « Les Sarkozy condamnés à la constipation?» reprend l'ensemble des procédés listés précédemment: jeux lexicaux relevant d'un registre familier, mots d'enfants, constructions syntaxiques diverses (présentatives, disloquées, averbales, inachevées...), ainsi 
que le rythme des enchaînements syntagmatiques qui s'apparente au rythme d'une narration orale et induit un effet de narration à rebondissements qui renforce le côté incongru et la visée satirique.

TERRIBLE! Après huit ans de procédure, voilà une bataille judiciaire perdue pour Sarkozy. Et non des moindres, puisqu'il s'agit d'une affaire d'État : celle des égouts du Cap Nègre. Le tribunal de Toulon a, le 28 mai, porté un coup sans doute fatal aux espérances de l'ancien couple présidentiel, qui bataillait pour voir ses déjections filer direct des WC au tout-à-l'égout. Sombre jour pour la République! [...] La légende veut que Carla Bruni-Sarkozy ait manqué de défaillir, à l'été 2008, en voyant du caca suinter d'un rocher. Vision répugnante due, selon elle, aux antiques fosses septiques. L'explication, selon ses adversaires, serait tout autre [...] Toujours est-il que Sarko, président, avait pris, si l'on ose dire, les choses en main et convoqué le préfet et le maire dans la propriété, les sommant de faire procéder d'urgence aux travaux. Le refus du préfet lui avait valu d'être viré [...] Hélas, trop sûrs d'eux en raison de ce prestigieux soutien, ses partisans ont négligé un détail : ils devaient organiser une assemblée générale extraordinaire de l'association syndicale et obtenir les quatre cinquièmes des votes. Impossible, à cause d'une poignée d'opposants! [...] En 2008, Sarkozy s'en vantait : «Affaire réglée». Bravo, maître Sarko!

Là encore, les effets d'oralité fonctionnent comme des modulations argumentatives structurantes. Le dédoublement dialogique du locuteur s'opère par l'alternance entre d'un côté, une énonciation objective et distanciée, qui se place dans un registre langagier et discursif académique, répondant aux contraintes de l'écrit journalistique couramment admises, et de l'autre côté, une énonciation plus idéologisée travaillée par ces effets d'oralité, et qui tend vers le discours indirect libre. Ce dédoublement fait en quelque sorte écho au double discours de mise dans l'univers politico-médiatique dénoncé, et c'est cet écho que permet la dynamique sarcastique.

Cette dynamique pragmatico-énonciative se retrouve dans Minute, comme le montre l'article suivant du 20 mars 2014 ("Taubira : l'oie blanche»).

Ça y est! Taubira l'a trouvé! Quoi? Le fusible! Christine Maugüé, sa directrice de cabinet, avait complètement oublié de lui dire que Nicolas Sarkozy était sur écoutes! Si c'est pas ballot quand même... Sa collaboratrice la plus proche l'aurait su le 26 février mais elle n'y aurait pas prêté garde avant le 11 mars... Ou elle aurait eu la tête ailleurs... [...] À la limite, c'est une chance que la presse ait révélé l'affaire car sinon, à l'heure qu'il est, dame Taubira n'en saurait toujours rien... Une sacrée chance que, le 7 mars, elle ait lu dans Le Monde ce que sa directrice de cabinet n'a pas fait, à moins qu'elle ne l'ait lu et n'ait pas fait le rapprochement avec les courriers qu'elle avait dans son tiroir... [...]

Christiane Taubira, c'est donc bien ce qu'elle disait, c'est l'oie blanche. Innocente comme l'enfant qui vient de naître, naïve, candide, vierge de tout péché fût-il un péché véniel comme le mensonge par omission. Elle n'a pas menti, elle ne savait pas. Elle n'a pas menti, elle a juste été «imprécise» et tout n'est que «malen- 
tendu». Elle n'a pas menti, elle a été trompée. Elle est victime [...] Elle n'a jamais voulu éviter que les délinquants soient incarcérés, c'est la droite qui lui fait systématiquement un procès en laxisme, comme ce ministre de droite qu'est Manuel Valls, et d'ailleurs elle ne va tarder à le prouver lors de la discussion de son projet de réforme pénale [...] Les affaires du PS qui traînent en longueur, à Marseille par exemple? Ce n'est que de la responsabilité des juges qui ont besoin de temps pour ficeler leurs dossiers [...] La mise sur écoute d'un avocat qui scandalise la profession quasi unanime? Il ne doit plus y avoir de profession protégée, rien d'autre. Et s'il y a des fuites dans la presse, c'est que celle-ci fait son travail. Taubira? Une oie blanche.

On peut ainsi relever les dispositifs similaires à ceux énoncés plus haut. Les phénomènes d'effets d'oralité relevant du dialogisme interlocutif (explicitement : «Ça y est! Taubira l'a trouvé! Quoi ? Le fusible!», «Taubira? Une oie blanche», ou plus implicitement : "Ou elle aurait eu la tête ailleurs... ») soulignent le souci de créer une connivence avec le lecteur, et ce, dès le premier énoncé. Ils s’ancrent aussi dans du dialogisme interdiscursif, discours indirect libre (souligné par les pointillés), métacommentaires axiologisés (soulignés par un trait continu) ou empathisés par le biais de «reconstructions empathiques » (double soulignement) ${ }^{16}$, qui posent la dissonance entre les discours des différents énonciateurs. Là encore, le dédoublement dialogique du locuteur marque une forme d'alternance entre un dire objectivant, issu d'une routine journalistique et un dire plus axiologisé qui donne alors une forte dynamique communicative au texte. Il nous semble s'inscrire au-delà de la distinction établie entre genres à «énonciation objectivisée » et genres à «énonciation subjectivisée » dans la presse (Moirand, 2000, p. 47), dans un entre-deux où finalement prime la volonté exacerbée (et revendiquée) de parler vrai. La récurrence de ces effets qui fonctionnent, on l'a vu, comme des indices pour repérer l'hétérogénéité énonciative, finit par opposer un parler institutionnel, donné peu ou prou comme sclérosé, et un parler vrai, celui d'un «journal satirique paraissant le mercredi » pour Le Canard enchaîné ou celui d'un «hebdomadaire politiquement incorrect» pour Minute.

Cette opposition entre ce qui serait un parlervrai et authentique vs. un parler institutionnel et sclérosé ne va pas sans poser des "auto-représentations» spécifiques pour chaque journal. Ainsi, Minute tend à « dénoncer les mots de l'autre, et à travers ses mots à stigmatiser l'énonciateur lui-même, souvent en tant qu'il est un énonciateur de la falsification et de la manipulation par le mensonge » (Krieg-Planque, 1999, p. 28), là où Le Canard enchaîné cherche un consensus normatif, éthique ou moral, sur lequel appuyer sa rhétorique et son

16. Pour des raisons de place, nous n'entrons pas ici dans le détail de certains de ces énoncés pouvant être perçus comme hybrides et opérant un glissement à la fois énonciatif, modal (entre les discours des différents énonciateurs et leurs prises en charge) et pragmatique (s'agit-il plus d’informer ici, de dénoncer ou de railler?). 
ethos. Pourtant, une même stratégie pragmatique se déploie dans ces titres et nous incite à nous placer, à nouveau, dans l'axe d'analyse d'A. Krieg-Planque :

La presse d'extrême droite n'est pas la seule à faire le choix d'un mode énonciatif caractérisé par l'implicite. Pour ce qui concerne la fréquence d'emploi de la marque «sic », on a observé que Le Canard enchaîné et, dans une moindre mesure, Charlie Hebdo étaient des concurrents sérieux de Rivarol, de National Hebdo, de Minute et de Présent [...] Dans un «sic» de Minute et dans un «sic» de Charlie Hebdo, il y a la même part d'implicite, le même plaisir du déchiffrage, de la devinette, et le même plaisir de l'entre-soi que produit le sentiment de la devinette réussie. Et pourtant, pour un lecteur donné, un «sic» du Canard enchaîné ou de Charlie Hebdo n'est pas perçu de la même manière qu'un «sic » trouvé dans la presse d'extrême droite [...] C'est que tout dépend, somme toute, dans cette délectation qui est puisée dans l'implicite, d'avec qui on prend son plaisir. (Krieg-Planque, 1999, p. 32)

\section{Double stratégie pragmatique déployée}

L'utilisation d'effets d'oralité répond ainsi à une problématique à la fois locale et globale. Du point de vue local, il s'agit de mettre en contraste des discours portés par des sources énonciatives différentes, qui s'opposent entre elles. Du point de vue global, le locuteur entre dans une double stratégie pragmatique : celle de sortir du rang et celle de former communauté. La première cherche à affranchir le discours des contraintes d'un écrit académique, pour se poser en instance de dévoilement; sans voile, sans filtre, le locuteur devient le garant d'un parler vrai et sa relation à l'autre, au tiers et à l'institution, est empreinte de conflictualité. Il construit un ethos en ce sens, on le verra. La seconde stratégie table sur une connivence avec le lecteur qui lui permette d'asseoir cet ethos, en jouant sur des phénomènes d'interlocution aussi bien que sur le registre du populaire. Il en ressort ici que le lien entre ethos et éthique agit en rouage important de cette énonciation. Ce lien se construit, certes, dans des titres de presse aux valeurs idéologiques très différentes, renvoyant aussi bien à des communautés discursives qu'à un rapport à la réalité extralinguistique souvent bien distincts, mais il œuvre à chaque fois comme moteur de la fonction communicative du texte.

\section{«Sortir du rang »: une instance de dévoilement}

Si la posture de dénonciation engagée par les deux journaux s'actualise par le jeu de différents dispositifs discursifs à l'intérieur de la matérialité textuelle, ces constructions sont en partie le fait d'effets d'oralité (mais aussi de processus figuraux que nous n'avons fait que suggérer ici). Ces effets entrent au service d'une scénographie spécifique aux incidences pragmatiques importantes car ils modulent la visée argumentative et l'idéologisation du discours 
(Maingueneau, 1999, p. 85 : «la scénographie est l'ensemble des stratégies discursives élaborées par le locuteur pour légitimer son discours face au lecteur»). Il s'agit de construire une posture singulière, une posture qui légitime l'acte de dénonciation. La stratégie discursive dynamique déploie alors la construction d'un ethos dont le propos vise le dévoilement de l'information. Cet ethos se construit à travers un univers de discours aux singularités langagières prégnantes qui concourent à dénoncer et impliquent que le locuteur s'attribue "une place discursive particulière, celle du collectif politique» (Brugidou, 2013, p. 90). Le locuteur produit une vision décalée, hors du cadre médiatique traditionnel. Se met alors en scène une dualité énonciative, dialogisée et polarisée, qui se constitue en fait social dans la mesure où elle pose une rupture entre deux conceptions institutionnelles différentes, celle du journal et celle de la sphère médiatico-politique dénoncée.

Cette mise en scène énonciative répond à une conflictualité latente avec laquelle elle entretient des rapports de réciprocité : la scénographie contribue à construire cette conflictualité et celle-ci contribue à alimenter la scénographie déployée dans ces discours et l'ethos d'instance de dévoilement non conformiste. Les effets d'oralité servent alors de moyen d'expression d'un point de vue (avéré ou non avéré) attribuable à un personnage (par le jeu du discours indirect libre ou du discours direct) ou au locuteur-énonciateur qui se place en situation de conflictualité. Le discours se construit et se structure alors sur la superposition de deux prises en charge énonciatives : d'abord, celle du journaliste de presse avec un ethos de médiateur de l'information objectif et distancié qui le légitime, puis la prise en charge du métacommentaire par un énonciateur différent, qui, lui, s'appuie sur un ethos indépendant, non assujetti à des contraintes formelles, rebelle à tout formatage institutionnel, sur le fond et la forme. Ces deux prises en charge sont portées par deux temporalités distinctes : la première y est essentiellement celle de l'actualité transmise, la seconde y apparaît plutôt comme décalée.

Les effets d'oralité, à l'interface de cette conflictualité latente, permettent d'opérer ce balancement. C'est cette dynamique pragmatique qui rend possible et repérable la posture de dénonciation. Fondamentalement co-énonciatifs, ils constituent un nouvel espace d'interprétation, en dehors des routines journalistiques, un espace où règne une connivence interlocutive qui n'hésite pas à jouer de ce rapport à la norme sociale, éthique ou morale.

\section{Former communauté, le registre du populaire}

Par cette co-construction, le lecteur pénètre dans l'univers de discours des deux journaux, un "entre-soi » de dénonciation. Le Canard enchaîné et Minute se positionnent ainsi au cœur de communautés discursives qu'ils ont eux-mêmes constituées pour construire une base socioculturelle/politique partagée avec 
le lecteur et diffuser l'information selon les exigences de leur contrat générique, mais en établissant leurs propres schèmes d'appréhension du monde médiatico-politique et en s'inscrivant dans une volonté de constitution d'un réseau qui sert de point d'appui à la visée informative.

Ce réseau tient de la communauté discursive, au sens large, telle que la définit Jean-Paul Bernié :

appelée à caractériser les fonctions et les fonctionnements du cadre [discursif] en prenant en compte ses relations avec diverses pratiques sociales externes [...], elle construit un cadre permettant de cerner, de transposer à des fins diverses les conditions sociales d'élaboration des savoirs et d'émergence des savoirs [...] une communauté constituée sur la base d'une pratique sociale quelconque (production de biens matériels ou de connaissances scientifiques, etc.) est une communauté discursive [...] la matérialité de ses pratiques est un critère déterminant. (Bernié, 2002, p.77-78)

Engagés dans une démarche coopérative, les membres d'une communauté finissent par développer un sentiment d'appartenance au groupe, formant un ensemble d'individus soudés à travers des textes par le lien socio-discursif qu'ils maintiennent. C'est cette gestion discursive que les deux hebdomadaires travaillent puisque le jeu interactionnel bâti sur la base de mécanismes dialogiques crée une connivence établie sur la base de l'assimilation de ces normes communes et avec une vectorialité revendiquée, celle de la dénonciation satirique, par laquelle la stigmatisation passe par des contenus explicites autant qu'implicites. Cette connivence conditionne le positionnement du journal : chacun produit un discours qui répond aux contraintes du genre, mais qui construit également de nouveaux codes identificateurs et par là-même une communauté discursive. Face à la dichotomie posée par la posture du « seul contre tous », le principe communautaire repose sur la tension avec les autres, les tiers absents que le locuteur disqualifie. L'extériorité de ce tiers critiqué est justement le facteur crucial qui met en œuvre l'idéologisation par la dialogisation du discours.

Les marques d'oral familier font signe vers une stratégie de fonctionnement en décalage voire de transgression par rapport aux normes des périodiques d'information journalistique. Dans l'imaginaire langagier, cette stratégie a pour incidence sociodiscursive l'émergence en filigrane du stéréotype du populaire. Le stéréotype relève d'un processus de catégorisation et de généralisation (Amossy et Herschberg-Pierrot, 2005, p. 27) qui tend à produire une vision simplifiée, voire déformée du réel. Ici, le registre de langage qui s’émancipe du langage standard de la presse instaure une dialectique sociale entre intériorité et extériorité à ladite communauté puisque les procédés linguistiques relevés s'avèrent bien souvent des marqueurs d'une oralité populaire dérogeant au côté académique du discours médiatique environnant. 


\section{Du populaire au populiste?}

En usant des procédés d'oralisation de l'écrit, le souci de «faire peuple», qui s'apparente à une «quête communautaire " (Taguieff, 1997, p. 33) tend en effet à faire pencher le discours journalistique vers un discours idéologiquement populiste (ou populisant, si l'on ose un néologisme).

Revenons brièvement sur le parcours lexical de «populisme» pour éclairer notre approche. Si le terme populiste revêt aujourd'hui une « diversité d'emplois et de sens» (Charaudeau, 2011a), portant cependant, le plus souvent, une visée de disqualification de l'objet ainsi désigné ${ }^{17}$, il désignait historiquement, à la fin du xıx siècle, un discours né d'un mouvement socialiste progressiste en Russie («narodnichestvo ») et, parallèlement, d'un mouvement rural auxÉtats-Unis, qui proclamaient tous deux un discours critique envers le capitalisme : "discours destiné aux classes populaires et moyennes, opposition proclamée aux “élites", capacité d'utiliser les moyens modernes de communication, volonté de mobiliser politiquement des citoyens excédés par le "système" " (Halimi, 1998, p. 117).

Ensuite, comme le décrit Pierre-André Taguieff :

Le terme «populisme» est redevenu, dans les années 1990, un terme péjoratif, de strict usage polémique. Sa signification oscille entre "fascisme» (tel qu'il est imaginé dans la culture antifasciste) et démagogie : un "populiste», dans le langage médiatique, est soit un «fasciste», soit un démagogue. Il s'ensuit que le «populisme» semble se définir soit par son orientation antidémocratique («fasciste»), soit par son allure pseudo-démocratique. (Taguieff, 1997, p. 5)

Cependant, certains chercheurs soulignent également l'acception potentiellement positive du terme, dans le cadre d'une conception assez large du populisme à lier avec la démocratie. En effet, « le peuple se situe au cœur de la vision sociétale et politique de tout mouvement populiste ; le concept de "communauté” occupe donc dans cette mesure une importance fondamentale» (Mény et Surel, 2002, p.11). A ce titre, « le populisme relève ensuite d'une rhétorique manichéenne fondée sur la célébration du peuple bon, juste, simple et "le rejet des élites corrompues, incompétentes et complices", qui ont "trahi” le peuple» (Ibid., p.12).

C'est dans ce dernier sens que les effets d'oralisation repérés dans notre corpus peuvent être rapprochés du populisme. Au-delà des clivages politiques

17. «Mot-valise, concept inexpérimenté, surgeon bâtard d'un “peuple” indécis, le populisme n'est réductible ni à un régime politique particulier ni à des contenus idéologiques fixes [...], un système défensif pour communautés fictives » (Taguieff, 1997, p.4). «Populiste est employé aussi bien par la droite que par la gauche pour stigmatiser le parti adverse ou pour se défendre contre la stigmatisation [...] dans les deux cas, le populiste c'est l'autre, qui pour s'exprimer emploierait une rhétorique simpliste et essentialisante. »(Charaudeau, 2011a, p.101). Mentionnons ici le titre évocateur de l'article de Serge Halimi (1998). «Le “populisme”, voilà l'ennemi ! ». 
et dans une vision binaire du monde, s'y érige en effet un discours de proximité par lequel les effets d'oralité inscrivent en langue et en discours la voix du peuple. Il se produit une sorte de mise en abyme dans le texte de la voix d'une instance qui ne dit pas son nom mais qui se pose en s'opposant à une élite ou à un système. En cela, l'effort de neutralisation du cadrage normatif de l'écrit journalistique de ce corpus nous apparaît comme une pratique communicative qui ne semble pas ressortir d'un discours de populisme politique en tant que tel mais plutôt d'un discours idéologiquement populisant, foncièrement inscrit dans le débat public. Il fait donc partie intégrante d'une pratique démocratique de la parole ${ }^{18}$ tout en participant à une dramatisation de celle-ci par ces procédés de médiation par le dire. L'espace de circulation que représentent ces deux médias infléchit ce processus.

\section{Réflexions conclusives}

Dans ce corpus de presse engagée (corpus choisi, de fait, pour sa polarisation), le cadre langagier, lorsqu'il comporte des caractéristiques d'oralité dans sa forme écrite, revêt une dynamique pragmatique voire argumentative et des enjeux au niveau macro du dire : dialogisation et idéologisation du discours, mise en scène d'une conflictualité. La discontinuité discursive d'une énonciation dialogisée mène ici au dévoilement, au parler vrai, et vise un positionnement hors du cadrage habituel du journalisme policé. On a abordé pour cela les principaux procédés d'oralité mobilisés, puis les mises en œuvre en discours de ces effets d'oralité et enfin la double stratégie pragmatique déployée. Ces procédés d'oralité deviennent des effets d'oralité, basculant de l'écheveau de la langue à celui du discours.

L'intentionnalité du locuteur devient alors un facteur déclenchant pour ces effets qui procurent au discours une nouvelle orientation modale et invitent à l'activation d'un système de pensée en dehors de l'énoncé lui-même. Ils constituent les nœuds de ce maillage interdiscursif et interlocutif. Chacun porté par son registre idéologique, le Canard enchaîné et Minute marquent ainsi à travers ces points de saillance, l'altérité du monde politico-médiatique et l'identification à une figure éthique (au sens d'ethos et de l'éthique) de «seul contre tous». À la conflictualité sous-jacente répond une forme de pugnacité. Au registre du populaire, parfois peut-être, l'écueil d'un élan populiste.

18. Nous partageons en cela les observations de P. Charaudeau (2011) : «Et comme le populiste prétend rendre au peuple son pouvoir de décision, son discours promet une rédemption par la libération du joug qu'imposent les élites et les appareils administrativo-politiques. Il appelle donc au rétablissement de la souveraineté populaire par une action directe, immédiate, courtcircuitant les institutions, illusion d'une promesse à laquelle font écho les mots d'ordre revendicatifs [...] Le populiste se pose en "sauveur" en maniant certaines "stratégies observables" : en tant que "représentant du peuple", il construit un "ethos d'authenticité", un "ethos de sincérité" et un "ethos de puissance" au "service de l'intérêt général, du bien du peuple".» 


\section{Références}

ABEILlÉ Anne, 2005, «Les syntagmes conjoints et leurs fonctions syntaxiques », Langages, $\mathrm{n}^{0} 160$, p. 42-66.

Amossy Ruth, Herschberg-Pierrot Anne, 2005, Stéréotypes et clichés, Paris, A. Colin. AuthIER-Revuz Jacqueline, 1984, «Hétérogénéité(s) énonciative(s) », Langages, nº 73 , p. 98-111.

AuthIER-Revuz Jacqueline, 1995, Ces mots qui ne vont pas de soi : boucles réflexives et non-coïncidences du dire, Paris, Larousse.

Bayle Ariane, Bombart Mathilde, Garnier Isabelle, BoIssiéras Fabienne éd., 2016, "L'âge de la connivence : lire entre les mots à l'époque moderne", Cahiers $d u$ GADGES, $\mathrm{n}^{\circ} 13$.

BERNIÉ Jean-Paul, 2002, "L'approche des pratiques langagières scolaires à travers la notion de communauté discursive : un apport à la didactique comparée? ", Revue française de pédagogie, $\mathrm{n}^{\circ}$ 141, p. 77-88.

BlanCHE-BenVENISTE Claire, 1996, "Trois remarques sur l'ordre des mots dans la langue parlée », Langue française, $\mathrm{n}^{0} 111$, p. 109-117.

BlanChe-BenVeniste Claire, 2007, «Corpus de langue parlée et description grammaticale de la langue », Langage et société, n¹21-122, p.129-141.

Blanche-Benveniste Claire, 2010, Approches de la langue parlée en français, Paris, Ophrys.

Blanche-BenVeniste Claire, Bilger Mireille, 1999, «Français parlé - oral spontané. Quelques réflexions», Revue française de linguistique appliquée, vol. IV, nº2, p. 21-30.

Bonnafous Simone, Temmar Malika, 2008, Analyse du discours et sciences humaines et sociales, Paris, Ophrys.

BRUGIDOU Mathieu, 2013, "L'institutionnalisation de la norme sociale entre stigmatisation et dénonciation », Langage et société, nº 144, p. 85-105.

Chabrol Claude, 2006, "Humour et médias», Questions de communication, nº10, p. 7-17.

CHAPUT Louise, 2009, "La variation stylistique en tant que procédé discursif dans les blogues journalistiques», Actes du colloque Le français parlé dans les médias : les médias et le politique, Lausanne / 2009, M. Burger, J. Jacquin et R. Micheli éd., 〈http://www.unil.ch/clsl/files/live/sites/clsl/files/shared/Actes_FPM_2009/ ChaputFPM20092.pdf> (consulté le 11 octobre 2016).

Charaudeau Patrick, 2011a, «Réflexions pour l'analyse du discours populiste», Mots. Les langages du politique, n 97, p. 101-116.

Charaudeau Patrick, 2011b, «Des catégories pour l'humour. Précisions, rectifications, compléments ", Humour et crises sociales. Regards croisés France-Espagne, M. D. Vivero éd., Paris, L'Harmattan, 2011, p. 9-43.

DAhlet Véronique, 2003, Ponctuation et énonciation, Guyane, Éditions Ibis Rouge.

DornA Alexandre, 2007, «Pistes pour une étude contextuelle du discours politique

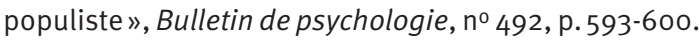


GADET Françoise, 1991, «Le parlé coulé dans l'écrit : le traitement du détachement par les grammaires du xxe siècle ", Langue française, $n^{\circ} 89$, p. 110-124.

Halımı Serge, 1998, "Le “populisme”, voilà l'ennemi !», Mots. Les langages du politique, $\mathrm{n}^{0} 55$, p. 115-121.

HeRmet Guy, Les populismes dans le monde. Une histoire sociologique, xIXe-xxe siècle, Paris, Fayard, 2001.

Kerbrat-Orecchioni Catherine, 1986, L'implicite, Paris, A. Colin.

KRIEG-PlAnqUE Alice, 1999, "Vacance argumentative : l'usage de (sic) dans la presse d'extrême droite contemporaine ", Mots. Les langages du politique, $\mathrm{n}^{\circ} 58$, p.11-34.

LE BART Christian, 1998, Le discours politique, Paris, PUF.

MaIngueneau Dominique, 1999, "Ethos, scénographie, incorporation », Images de soi dans le discours. La construction de l'ethos, R. Amossy éd., Lausanne, Delachaux et Niestlé, p. 75-100.

Maingueneau Dominique, 2002, "Problèmes d'ethos», Pratiques, n¹13-114, p. 55-68.

MARTIN, Laurent, 2003, "Le Canard enchaîné, un "objet politique mal identifié" ", Revue d'histoire moderne et contemporaine, $\mathrm{n}^{\circ}$ 2, p. 73-91.

Mény Yves, SuRel Yves, 2000, Par le peuple, pour le peuple. Le populisme et les démocraties, Paris, Fayard.

Mény Yves, Surel Yves éd., 2002, Democracies and the Populist Challenge, Londres, Palgrave.

MoIRAND Sophie, 2000, «Variations discursives dans deux situations contrastées de la presse ordinaire», Les Carnets du Cediscor, n 6, p. 45-62.

NeVeu Érik, 1993, "Pages "Politique” », Mots. Les langages du politique, n³7, p. 6-27.

NowAKowsKA Aleksandra, "Thématisation et dialogisme : le cas de la dislocation. », Langue française, n 163, p.79-98.

Peeters Bert, 2007, "Effets d'oralité dans un corpus de faits divers de langue française », Le français parlé des médias, M. Broth, M. Forsgren, C. Norén, F. SulletNylander éd., Stockholm, Université de Stockholm, p. 609-623 («Acta Universitatis Stockholmiensis, Romanica Stockholmiensia », 24).

PERRIN Laurent, 2009, "L'ethos de l'oralité comme forme de fiction énonciative dans la presse écrite", Actes du colloque Le français parlé dans les médias : les médias et le politique, M. Burger, J. Jacquin, R. Micheli éd., Lausanne, 2009, 〈http://www.unil. ch/clsl/files/live/sites/clsl/files/shared/Actes_FPM_2009/PerrinFPM2009.pdf> (consulté le 11 octobre 2016).

RABATELAlain, 2006, "L'effacement de la figure de l'auteur dans la construction évènementielle d'un "journal” de campagne électorale et la question de la responsabilité en l'absence de récit primaire », Semen, n²2, p. 77-92.

RABATEL Alain, 2011, «Les paradigmes entrecroisés des instances énonciatives et des points de vue », Naissance d'un paradigme, Actes du XVIIe séminaire international de didactique universitaire de Constanta (Roumanie) 26 août-1er septembre 2010, F. Hrubaru, E. Comes éd., [Bucarest], Association des chercheurs en linguistique française (ACLIF), p. 9-28.

Rabatel Alain, 2013, "Écrire les émotions en mode empathique», Semen, nº 35 , p. $65-82$ 
ROSIER Laurence, 2000, «Interjection, subjectivité, expressivité et discours rapporté à l'écrit : petits effets d'un petit discours », Cahiers de praxématique, $\mathrm{n}^{\circ} 34$, Montpellier, PULM, p. 19-50.

SeoAne Annabelle, 2015, "Quand le Canard enchaîné médit sans (vraiment) dire», Semen, n 40, p. 91-109.

SPerber Dan, Wilson Deirdre, 1978, «Les ironies comme mention », Poétique, n 36, p. 399-412.

TAGUieff Pierre-André, 1997, «Le populisme et la science politique du mirage conceptuel aux vrais problèmes ", Vingtième siècle, nº 56, p. 4-33.

TAguieff Pierre-André, 2007, L'illusion populiste : essai sur les démagogies de l'âge démocratique, Paris, Flammarion.

TUOMARLA, Ulla, 1999, «Le discours direct dans la presse écrite : Un lieu de l'oralisation de l'écrit», Faits de langues, nº13, p. 219-229. 